\title{
Espiritualidade na formação acadêmica em psicologia no Rio Grande do Sul
}

\author{
Spirituality in psychology academic training: A \\ comparative study of senior and freshmen \\ undergradute students in Rio Grande do Sul
}

\author{
Carla Maria Frezza CAVALHEIRO \\ Denise FALCKE ${ }^{2}$
}

\begin{abstract}
Resumo
Embora estudos científicos recentes comprovem correlação positiva entre espiritualidade e saúde, outros apontam a espiritualidade do psicólogo como menor que a da população em geral. Visando analisar a espiritualidade de acadêmicos de psicologia, foram investigados 1064 estudantes (672 calouros e 392 formandos) de todas as universidades gaúchas com formandos em 2009. Foram aplicados questionário biossociodemográfico, questionário sobre valores e aspectos espirituais e religiosos, Escala de Bem-Estar Espiritual e Subescala de espiritualidade, religiosidade e crenças pessoais do World Health Organization Quality of Life Group-100. Os resultados revelaram que formandos apresentam índices significativamente menores de Bem-Estar Espiritual $(t=3,769 ; p<0,001)$. Eles também referem acreditar significativamente menos em Deus, força superior e/ou energia $\left(\chi^{2}=10,03 ; p<0,001\right)$. Além disso, a importância da espiritualidade na clínica psicológica e no enfrentamento de situações cotidianas também é menor para os formandos $(p<0,001)$. Esses dados indicam que provavelmente o curso de psicologia contribua para o declínio da espiritualidade, o que revela a necessidade de reavaliação sobre como a espiritualidade está sendo abordada na graduação.
\end{abstract}

Palavras-chave: Bem-estar espiritual; Espiritualidade; Formação acadêmica; Psicologia transpessoal.

\begin{abstract}
Many studies discuss the positive correlation between spirituality and health and some highlight the fact that the psychologists' spirituality is significantly lower than that of the population. This study aimed to analyze the level of spirituality of 1,064 psychology students (672 freshmen and 392 senior students) from all universities in the State of Rio Grande do Sul. The instruments used included a sociodemographic questionnaire, a questionnaire covering spiritual and religious aspects and values, the Spiritual Well-Being Scale, and the Spirituality, Religiousness and Personal Beliefs Sub-scale of the World Health Organization Quality of Life -100 instrument. The results revealed that the senior
\end{abstract}

$\boldsymbol{\nabla} \boldsymbol{\nabla} \boldsymbol{\nabla}$

1 Universidade de Caxias do Sul, Centro de Ciências Sociais Aplicadas, Curso de Administração. R. Francisco Getúlio Vargas, 1130, 95070-560, Caxias do Sul, RS, Brasil. Correspondência para/Correspondence to: C.M.F. CAVALHEIRO. E-mail: <holospsi@terra.com.br>.

2 Universidade do Vale do Rio dos Sinos, Centro de Ciências da Saúde, Programa de Pós-Graduação em Psicologia Clínica. São Leopoldo, RS, Brasil.

Artigo elaborado a partir da dissertação de C.M.F. CAVALHEIRO, intitulada "Espiritualidade na clínica psicológica: um olhar sobre a formação acadêmica no Rio Grande do Sul”. Universidade do Vale do Rio dos Sinos, 2010. 
students had significantly lower levels of spiritual well-being ( $t=3.769 ; p<0.001)$. They believed in God, a superior being and/or energy, significantly less frequently than the freshmen $\left(\chi^{2}=10.03 ; p<0.001\right)$. Likewise, for the senior students the importance of spirituality in the psychological practice and in coping with day-to-day situations was always indicated as significantly lower than for the freshmen $(p<0.001)$. These data indicate that the undergraduation in psychology possibly contributes to a decline in spirituality among its students, highlighting the need to reassess how spirituality is being approached in the undergraduate psychology courses.

Keywords: Spiritual well-being; Spirituality; Academic education; Transpersonal psychology.

As transições paradigmáticas refletiram, em muitos momentos, uma intensa dicotomia entre ciência e religião. Nesse sentido, durante muito tempo, os estudos científicos sobre espiritualidade, religião e religiosidade foram escassos nas diferentes áreas do conhecimento, em especial na psicologia. Nas últimas décadas, entretanto, tem-se observado aumento significativo da produção científica sobre o tema. Consulta realizada na base de dados PsycINFO, por Koenig (2007), aponta que, da década de 1970 para a primeira década do século XXI, houve aumento de $600 \%$ no número de artigos que tinham como descritores as palavras "religion", "religiosity", "religious beliefs" e "spirituality". De forma complementar, Aten e Hernandez (2005) publicaram um artigo no qual apresentam uma revisão de 2726 publicações, de 1978 a 2003, em 7 diferentes jornais e revistas voltados para psicologia e espiritualidade.

Resultados de pesquisas recentes (Costa et al., 2008; Koenig, 2007; Marques, 2000, Panzini, Rocha, Bandeira, \& Fleck, 2007; Pedrão \& Beresin, 2009; Peres, Simão, \& Nazello, 2007) apontam para o fato de que a literatura científica tem apresentado a existência de relação significativa entre saúde, qualidade de vida e espiritualidade. Estudos atuais também investigam o quanto a espiritualidade pode auxiliar no enfrentamento de situações estressantes e traumáticas (Panzini et al., 2007), na promoção de qualidade de vida (Panzini et al., 2007), na relação com a saúde geral (Marques, 2003), em contextos de violência e desestruturação familiar, no tratamento e na prevenção da dependência química e no abandono escolar (Sanchez \& Nappo, 2007), nas questões referentes à sexualidade de jovens (Cerqueira, 2008), no acompanhamento de pacientes crônicos e terminais (Kovács, 2007), no desenvolvimento da resiliência (Rocca-Larrosa, 2007), nos

36 transtornos psicóticos (Koenig, 2007; Lukof, 2003), nos riscos de suicídio (Moreira-Almeida, Lotufo Neto, \& Koenig, 2006) e até no diagnóstico diferencial entre psicose e emergência espiritual, que passa a ser necessário a partir da inclusão desta categoria de emergência espiritual no DSM-IV (Diagnostic and Statistical Manual of Mental Disorders - Fourth Edition) (Lukoff, 2003). Além disso, existem estudos focados especificamente na espiritualidade dos psicólogos (Marques, 1996; Peres et al., 2007), na espiritualidade dos estudantes durante a formação acadêmica em psicologia (Costa et al., 2008; Gastaud et al., 2006) e, principalmente, na importância da espiritualidade na clínica psicológica (Ancona-Lopez, 2008a; Angerami-Camon, 2008; Boccalandro, 2004; Bruscagin, Savio, Fontes, \& Gomes, 2008; Erthal, 2004).

O bem-estar psicológico e espiritual e as implicações clínicas dessa relação foram amplamente avaliados por Moreira-Almeida, Lotufo Neto e Koenig (2006), em revisão que teve como base 850 artigos sobre o tema. Segundo os autores, estudos relevantes e confiáveis apontaram que maiores níveis de envolvimento espiritual estão positivamente associados a indicadores de bem-estar psicológico, tais como: satisfação com a vida, felicidade, afeto positivo e moral mais elevado. Além disso, demonstram associações negativas entre espiritualidade e depressão, comportamento suicida, uso ou abuso de álcool e drogas.

No entanto, enquanto dados estatísticos revelam que, em linhas gerais, mais de três quartos dos americanos acreditam na vida após a morte física e, portanto, na dimensão espiritual, e que cerca de $90 \%$ da população em geral acreditam em Deus, apenas $43 \%$ de uma amostra de psicólogos e psiquiatras, membros da American Psychological Association (APA), têm tal crença (Greeley \& Hout, 1999). Da mesma forma, estudantes de psicologia 
europeus demonstraram preconceito diante da questão da espiritualidade e sua relação com a saúde mental (Gastaud et al., 2006).

No Brasil, segundo o Instituto Brasileiro de Geografia e Estatística (2000), apenas 7,3\% da população não têm nenhuma religião, e 92,0\% da população em geral levam em consideração as questões espirituais. Segundo Ancona-Lopez (1999), em média, 90,0\% dos clientes de psicoterapia identificam-se com alguma religião, 86,0\% acreditam em Deus, 49,0\% frequentam alguma instituição religiosa e $47,0 \%$ consideram a fé muito importante em suas vidas, o que torna relevante o questionamento sobre a dimensão espiritual dos próprios psicólogos.

Estudo realizado por Gastaud et al. (2006), que investigou a relação entre bem-estar espiritual e transtornos psiquiátricos menores em acadêmicos de psicologia, medicina e direito, revelou que, entre os alunos de psicologia, 84,6\% apresentaram índices de espiritualidade significativamente baixos, enquanto nos cursos de medicina e direito, baixos índices de espiritualidade foram atribuídos a 68,0\% dos estudantes.

Apesar desses baixos índices, o resultado de um estudo realizado por Costa et al. (2008), com 136 universitários de psicologia da Pontifícia Universidade Católica do Rio Grande do Sul (PUCRS), verificou que existe correlação positiva e altamente significativa entre bem-estar existencial e psicológico desses universitários. Os achados do presente estudo confirmam a importância de o tema ter uma inserção consolidada na formação dos universitários, pois $86,0 \%$ dos participantes referiram ter religião, 83,8\% têm interesse pelo assunto espiritualidade e $77,9 \%$ têm interesse em discutir o assunto na sua formação.

Sendo assim, torna-se imprescindível investigar quanto o curso de Psicologia pode estar contribuindo para os baixos índices de espiritualidade de estudantes e profissionais da área. Assim sendo, o principal objetivo desta pesquisa é avaliar a espiritualidade de estudantes de Psicologia do Rio Grande do Sul, ou, mais especificamente:

- investigar os índices de bem-estar espiritual, nas dimensões religiosa e existencial, e a dimensão espiritualidade, religiosidade e crenças pessoais de estudantes do curso de Psicologia das universidades gaúchas;

- comparar os índices de espiritualidade dos calouros e formandos em Psicologia no Rio Grande do Sul;

- verificar as possíveis diferenças nos níveis de espiritualidade com relação às linhas teóricas com as quais os formandos mais se identificam.

\section{Método}

\section{Participantes}

A amostra foi composta por 1064 estudantes das 25 universidades do Rio Grande do Sul que tiveram formandos em Psicologia no segundo semestre de 2009. Ao todo, foram 672 calouros $(63,2 \%)$ e 392 formandos (36,8\%). O perfil da amostra (Tabela 1) descreve os subgrupos de calouros e formandos que participaram do estudo. $O$ critério para inclusão na amostra desta pesquisa foi que os alunos tão somente estivessem matriculados e presentes em sala de aula, nas disciplinas que tinham maior número de calouros ou formandos em cada universidade quando da coleta dos dados (Tabela 1).

\section{Instrumentos}

Questionário de identificação biossociodemográfica e econômica, composto por 10 questões que avaliaram idade, sexo, universidade em que estuda, semestre do curso, situação ocupacional, renda familiar, estado civil, número de filhos, presença de alguma doença crônica e como o participante avalia seu estado de saúde.

Questionário sobre valores e aspectos espirituais e religiosos, elaborado para este estudo: composto por 21 questões sobre aspectos espirituais e religiosos (tais como crença em Deus, prática de alguma religião, mudança de religião/doutrina/ crença ao longo da vida, se a crença espiritual é a mesma dos familiares, com que frequência o parti- 
Tabela 1

Descrição das características da amostra. São Leopoldo (RS), 2010

\begin{tabular}{|c|c|c|c|c|}
\hline \multirow{2}{*}{ Variáveis } & \multicolumn{2}{|c|}{ Calouros } & \multicolumn{2}{|c|}{ Formandos } \\
\hline & $\%$ & $n$ & $\%$ & $n$ \\
\hline \multicolumn{5}{|l|}{ Sexo } \\
\hline Homens & 15,3 & 103 & 10,7 & 42 \\
\hline Mulheres & 84,4 & 567 & 88,8 & 342 \\
\hline Não responderam & 0,3 & 2 & 0,5 & 2 \\
\hline \multicolumn{5}{|l|}{ Situação Conjugal } \\
\hline Solteiro & 79,3 & 532 & 65,6 & 257 \\
\hline Casado ou união estável & 17,8 & 99 & 30,3 & 119 \\
\hline Separado/divorciado & 2,7 & 18 & 5,1 & 16 \\
\hline Viúvo & 0,3 & 2 & 0 & 0 \\
\hline \multicolumn{5}{|l|}{ Filhos } \\
\hline Sem filhos & 81,4 & 546 & 76,4 & 298 \\
\hline 1 filho & 10,9 & 73 & 10,5 & 41 \\
\hline 2 filhos & 4,9 & 33 & 9,0 & 35 \\
\hline 3 filhos & 2,5 & 17 & 3,1 & 12 \\
\hline 4 filhos & 0 & 0 & 1,0 & 4 \\
\hline \multicolumn{5}{|l|}{ Renda } \\
\hline Até 5 salários-mínimos & 49,2 & 303 & 29,0 & 106 \\
\hline Entre 5 e 10 salários-mínimos & 30,5 & 188 & 38,0 & 139 \\
\hline Entre 10 e 20 salários-mínimos & 14,1 & 87 & 23,2 & 85 \\
\hline Mais de 20 salários-mínimos & 6,2 & 38 & 9,8 & 36 \\
\hline \multicolumn{5}{|l|}{ Situação Ocupacional } \\
\hline Só estudam & 49,6 & 331 & 46,5 & 182 \\
\hline Estudam e têm trabalho remunerado & 44,2 & 295 & 32,2 & 126 \\
\hline Estudam e têm trabalho não remunerado & 6,3 & 42 & 21,2 & 83 \\
\hline
\end{tabular}

cipante vai à igreja, templo ou encontros de natureza religiosa, com que frequência dedica-se à oração, meditação ou leituras sagradas, quão importante considera a espiritualidade para lidar com as situações estressantes e conflitivas, quão importante considera a espiritualidade no processo de autoconhecimento, na qualidade de vida ou na saúde de forma global). Além dessas, foi proposta uma questão que apresentava dez valores em ordem alfabética (amizade, conhecimento, dinheiro, espiritualidade, família, lazer, relacionamento amoroso, saúde, sexo e trabalho), para serem elencados conforme as prioridades e o grau de importância atribuído a cada um deles. A análise de confiabilidade do instrumento, considerando as questões propostas a partir de uma escala likert de cinco pontos, revelou bom nível de consistência interna, com a obtenção de coeficiente alpha de 0,873.

Escala de Bem-Estar Espiritual (EBE), versão 38 em português da Spiritual Well-Being Scale, criada por Paloutzian e Ellison, adaptada e validada para o Brasil por Marques, Sarriera e Dell'Aglio (2008). A EBE é composta por 20 questões com seis alternativas de resposta para cada uma delas. Do total, 10 avaliam o Bem-Estar Religioso (BER), considerado como advindo da relação íntima com Deus, e as outras 10, o Bem-Estar Existencial (BEE), que seria relativo à satisfação geral com a vida (Paloutzian \& Elisson, 1982). Os escores das subescalas do Bem-Estar Existencial e religioso são somados para obtenção da média geral de Bem-Estar Espiritual (EBE). Testes de confiabilidade, realizados para a versão em português, obtiveram coeficientes alfa de 0,92 para o índice geral (EBE), 0,92 (BER) e 0,85 (BEE). No presente estudo, os índices encontrados foram 0,901 (EBE), 0,922 (BER) e 0,790 (BEE).

Dimensão espiritualidade, religiosidade e crenças pessoais Spirituality, Religiousness and Personal Beliefs (SRPB) do WHOQOL-100 (WHOQOL Group, 1994): a dimensão é composta por quatro das 100 questões do instrumento. Na sua íntegra, o WHOQOL-100 teve teste de campo realizado em 20 países, apresentando boa consistência interna (coeficiente alpha de Cronbach de todas as subescalas acima de 0,5) (WHOQOL Group, 1994). Neste estudo, o coeficiente da dimensão SRPB foi de 0,839 .

\section{Procedimentos}

Inicialmente, foram contatados todos os cursos de psicologia do estado para a devida identificação das universidades que teriam formandos no segundo semestre de 2009. Posteriormente, foi estabelecido contato com a coordenação dos cursos de psicologia e solicitada autorização para apresentação do projeto e realização do estudo. Em seguida, após aprovação do Comitê de Ética da Universidade do Vale do Rio dos Sinos (Parecer $n^{\circ}$ 09/003), retomou-se o contato com as coordenações dos cursos por e-mail e por telefone. Foram identificadas, em cada universidade, as disciplinas nas quais estava matriculado o maior número de calouros e formandos, e a coleta foi realizada em sala de aula, com exceção de apenas uma universidade, entre as 25 pesquisadas, em que a coleta 
foi realizada em horário extraclasse. Após assinarem o termo de consentimento livre e esclarecido, os estudantes que aceitaram participar da pesquisa responderam aos questionários, cujo preenchimento durou de 30 a 45 minutos nos diferentes grupos.

Os dados foram analisados pelo programa estatístico SPSS (versão 18.0).

\section{Resultados e Discussão}

No presente estudo, para avaliação dos índices de espiritualidade, foram utilizados dois instrumentos: EBE e SRPB/WHOQOL-100. Assim sendo, doravante, índices de espiritualidade se referem aos resultados obtidos pelos dois instrumentos; do contrário, utilizaremos a denominação de cada instrumento.

Avaliando-se os índices de EBE de estudantes do curso de Psicologia das universidades gaúchas, verifica-se que a média obtida por esses acadêmicos foi de 90,04 ( $D P=16,34)$, com variação entre 32 e 120. Considerando-se as subescalas referentes ao Bem-Estar Existencial e Religioso, observa-se que a média de BEE foi de 46,00 ( $D P=7,53)$, e a de $B E R$ foi de $44,00(D P=11,42)$. Como a escala não tem um ponto de corte definido, a análise desses índices só é possível a partir da comparação com outros estudos que utilizaram o mesmo instrumento.

Ao comparar os níveis de bem-estar espiritual dos estudantes de psicologia do Rio Grande do Sul obtidos neste estudo com os de outros estudos que também utilizaram a EBE, seja com estudantes de direito, medicina, profissionais de enfermagem, seja com a população em geral, evidencia- -se uma menor pontuação na EBE. A única exceção foi o estudo realizado por Costa et al. (2008), com acadêmicos de psicologia da PUCRS, que encontrou OS seguintes escores: $E B E=87,95(D P=15,37)$; $B E R=41,73(D P=10,86)$ e $B E E=46,51 \quad(D P=7,85)$. Muito provavelmente, os índices de EBE do presente estudo $(90,04)$ podem ser atribuídos à preponderância de calouros $(63,15 \%)$, enquanto no estudo da PUC, os alunos estiveram igualmente representados do $1^{\circ}$ ao $10^{\circ}$ semestre da formação em psicologia.

A dimensão de espiritualidade, religiosidade e crenças pessoais SRPB do WHOQOL - 100 obteve a média de 15,74 ( $D P=2,84)$, com variação entre 5 e 20. Como não foram identificados outros estudos que tenham feito uso isolado da dimensão SRPB até o momento, não foi possível realizar comparativos com esses dados.

\section{Comparação dos índices de espiritualidade de calouros e formandos}

Na comparação entre calouros e formandos, por meio do teste $t$ de Student, foram encontradas diferenças significativas nos índices de EBE (Tabela 2).

Os dados da tabela revelam diferenças significativas entre calouros e formandos nas dimensões de Bem-Estar Religioso $(p<0,001)$ e Bem-Estar Espiritual $(p<0,001)$, sendo a média obtida pelos calouros maior do que a dos formandos em todas as dimensões. Sendo a EBE o somatório de BEE e BER, verifica-se que a dimensão religiosa (crença em Deus) interferiu na diminuição dos índices de Bem-Estar

Tabela 2

Calouros e formandos: níveis de Bem-estar Espiritual. São Leopoldo (RS), 2010

\begin{tabular}{|c|c|c|c|c|c|c|}
\hline \multirow{2}{*}{ Variável } & \multicolumn{2}{|c|}{ Calouros } & \multicolumn{2}{|c|}{ Formandos } & \multirow{2}{*}{$t$} & \multirow{2}{*}{ Valor de $p$} \\
\hline & M & DP & M & DP & & \\
\hline Bem-estar Religioso & 45,48 & 11,15 & 41,46 & 11,42 & 5,458 & $<0,001$ \\
\hline Bem-estar Existencial & 46,04 & 7,53 & 45,92 & 7,52 & 0,245 & 0,807 \\
\hline Bem-estar Espiritual & 91,51 & 16,31 & 87,49 & 16,07 & 3,769 & $<0,001$ \\
\hline Religiosidade/Espiritualidade e crenças pessoais & 15,83 & 2,96 & 15,58 & 2,61 & 1,395 & 0,177 \\
\hline
\end{tabular}

Nota: M: Média; DP: Desvio-Padrão. 
Espiritual (EBE). Dessa forma, a partir dos resultados deste estudo, pode-se inferir que o curso de psicologia interfere nos níveis de Bem-Estar Religioso e Espiritual dos acadêmicos, que são menores nos formandos em comparação aos calouros.

Pode-se observar que, quando comparados os calouros e os formandos de psicologia, não foram apontadas diferenças significativas na dimensão SRPB-WHOQOL 100 ( $t=1,395 ; p=0,177)$. Como a SRPB tem uma concepção de espiritualidade que se aproxima da subescala de bem-estar existencial da $E B E$, acentua-se a hipótese de que a diferença entre calouros e formandos é centrada no aspecto religioso, ou seja, no declínio da crença em Deus.

Por meio dos dados do questionário sobre valores e aspectos espirituais e religiosos, essa diferença também é confirmada. Ao serem questionados se acreditam em Deus (considerado como poder, espírito, força superior, inteligência suprema, consciência cósmica, energia divina, entre outros), $93,1 \%$ dos calouros e $87,2 \%$ dos formandos responderam que sim, caracterizando uma diferença significativa entre os grupos $\left(\chi^{2}=10,03 ; p=0,001\right)$. Comparando esse dado com o da população brasileira em geral - segundo O IBGE (2000), apenas $7,0 \%$ das pessoas não acreditam em Deus -, perceber-se que a formação em Psicologia parece contribuir para a descrença em Deus, uma vez que essa crença presente nos calouros, que é igual à da população em geral, declina significativamente ao final da graduação.
A pontuação na escala de valores quanto ao que teria maior e menor prioridade também apresentou diferença significativa entre calouros e formandos $(p<0,001)$. Considerando os valores pontuados como de maior prioridade, a espiritualidade foi referida por $13,1 \%$ dos calouros e somente por $6,2 \%$ dos formandos. Entretanto, considerando os valores pontuados como de menor prioridade, a espiritualidade foi referida por $17,7 \%$ dos calouros e por $27,8 \%$ dos formandos.

Em relação à espiritualidade como forma para lidar com situações da vida pessoal, também foram observadas diferenças significativas entre os grupos ( $t=3,654 ; p<0,001): 73,2 \%$ dos calouros a consideram importante ou extremamente importante, enquanto $56,6 \%$ dos formandos apresentaram essa opinião. Os dados relativos à possível influência da espiritualidade no processo de autoconhecimento, à qualidade de vida e à saúde mental estão na Tabela 3.

Uma vez que a análise estatística revelou a existência de diferenças significativas nos três itens $(p<0,001)$, verifica-se que, em comparação aos calouros, o número de formandos que acredita que a espiritualidade "não influencia" o autoconhecimento, a qualidade de vida e a saúde mental das pessoas é significativamente maior. Todavia, é importante ressaltar que calouros e formandos avaliam preponderantemente a influência negativa ou muito negativa da espiritualidade nas dimensões estudadas, evidenciando desconhecimento dos estudantes diante das pesquisas que já comprovaram

\section{Tabela 3}

Influência atribuída à espiritualidade por calouros e formandos. São Leopoldo (RS), 2010

\begin{tabular}{|c|c|c|c|c|c|c|}
\hline$\stackrel{n}{3}$ & Influencia & $\begin{array}{c}\text { Influencia muito } \\
\text { negativamente }\end{array}$ & $\begin{array}{c}\text { Influencia } \\
\text { negativamente }\end{array}$ & $\begin{array}{c}\text { Não } \\
\text { influencia }\end{array}$ & $\begin{array}{c}\text { Influencia } \\
\text { positivamente }\end{array}$ & $\begin{array}{l}\text { Influencia muito } \\
\text { positivamente }\end{array}$ \\
\hline 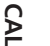 & \multicolumn{6}{|l|}{ Autoconhecimento } \\
\hline 盂 & Calouros & 34,3 & 47,9 & 16,4 & 0,9 & 0,5 \\
\hline O & Formandos & 20,4 & 50,9 & 27,1 & 1,6 & 0,0 \\
\hline ְ. & \multicolumn{6}{|l|}{ Qualidade de vida } \\
\hline \multirow{4}{*}{$\begin{array}{l}\text { 离 } \\
\text { 侖 }\end{array}$} & Calouros & 36,2 & 49,0 & 14,2 & 0,3 & 0,3 \\
\hline & Formandos & 21,2 & 49,7 & 27,7 & 1,3 & 0,0 \\
\hline & Saúde mental & & & & & \\
\hline & Calouros & 37,4 & 48,5 & 13,2 & 0,7 & 0,1 \\
\hline 40 & Formandos & 21,1 & 52,9 & 22,1 & 2,6 & 1,3 \\
\hline
\end{tabular}


uma correlação positiva entre espiritualidade e saúde (Costa et al., 2008; Fleck, 2004; Koenig, 2007; Marques, 2000, 2003; Panzini et al., 2007; Pedrão \& Beresin, 2009; Peres et al., 2007). O fato torna-se ainda mais grave no caso dos formandos, uma vez que, durante a formação, eles deveriam ter tido acesso aos estudos científicos contemporâneos que tratam do tema.

A fim de aprofundar a compreensão sobre a relação entre espiritualidade e psicologia, questionaram-se os estudantes sobre o quão importante eles consideram a dimensão espiritual para a prática clínica da psicologia. Os resultados também evidenciaram diferenças significativas entre calouros e formandos ( $t=3,295 ; p<0,001)$ : uma porcentagem maior de formandos $(69,3 \%)$ do que de calouros $(46,2 \%)$ considera que ela seja pouco ou nada importante. Somente $13,4 \%$ de calouros e $4,2 \%$ de formandos consideraram a espiritualidade extremamente importante para a prática clínica em psicologia. Peres et al. (2007) referem que psicoterapia, espiritualidade e religiosidade são temas altamente relacionados e enfatizam que a importância do estudo do papel da espiritualidade em relação às práticas terapêuticas se justifica tanto por razões socioeconômicas como clínicas. No Brasil, no entanto, ainda são escassas tanto as abordagens quanto os psicoterapeutas que contemplam a religiosidade e a espiritualidade.

Assim, encontraram-se fortes indícios de que a formação acadêmica em psicologia não vem propiciando aos seus acadêmicos o devido conhecimentos das interfaces entre espiritualidade e prática clínica, conforme já apontam estudos atuais realizados por outros pesquisadores (Ancona-Lopez, 2008b; Angerami-Camon, 2008; Boccalandro, 2004; Bruscagin et al., 2008).

\section{Espiritualidade em relação às linhas teóricas de maior identificação dos formandos}

No instrumento de pesquisa, algumas questões foram dirigidas exclusivamente aos formandos; uma delas questionava sobre sua possível identificação com as linhas teóricas estudadas na for- mação em Psicologia. Considerando os formandos que responderam a essa questão, verifica-se que 60,5\% têm maior identificação com a Psicanálise, 24,1\% com a Cognitivo-Comportamental, 5,5\% com a Sistêmica, 3,2\% com a Gestalt, 2,3\% com a Humanista, 2,3\% com a Psicologia Social Crítica, 0,9\% com a Esquizoanálise, 0,5\% com a Junguiana, 0,5\% com a Fenomenológica e 0,5\% disseram se identificar com várias abordagens. Para fins de realização da Anova, optou-se por agrupar as abordagens em três grupos: 1) Psicanálise; 2) Cognitivo-Comportamental 3) Humanismo, Gestalt, Sistêmica, Junguiana e Fenomenológico-Existencial. As outras abordagens citadas, por apresentarem baixa incidência e características peculiares que dificultam o agrupamento com as demais, foram desconsideradas nesta análise. Comparando os três grupos, não se observa diferença significativa nas dimensões do Bem-Estar Religioso ( $F=2,043 ; p=0,132)$ e do Bem-Estar Existencial $(F=1,627 ; p=0,199)$. Contudo, na dimensão de Bem-Estar Espiritual, há uma diferença significativa $(F=3,061 ; p=0,049)$, e, na dimensão SRPB, verifica-se uma tendência à diferenciação entre os grupos ( $F=2,899 ; p=0,057)$. As médias da dimensão de Bem-Estar Espiritual revelam que o grupo com maiores níveis foi o três: Humanismo, Gestalt, Sistêmica, Junguiana e Fenomenológico Existencial $(M=90,75 ; D P=16,43)$, seguido proximamente pelo dois: Cognitivo Comporamental ( $M=90,72$; $D P=15,26)$, e, com os menores índices de Bem-Estar Espiritual, o grupo um: Psicanálise $(\mathrm{M}=85,18 ; \mathrm{DP}=15,54)$.

$\mathrm{O}$ alto percentual de formandos que dizem ter estudado predominantemente a psicanálise, em detrimento de outras escolas pouco abordadas durante a formação, pode ser um fator que influencie a consequente eleição dessa abordagem como sua opção de linha teórica, por ser mais provável identificar-se com o que se tem maior conhecimento. Sendo os formandos que se dizem identificados com a psicanálise justamente os que apresentam os menores índices de espiritualidade, parece necessária uma revisão sobre o quão dogmáticos seriam os postulados da teoria psicanalítica diante da religiosidade e da espiritualidade humana, bem como o quanto esses postulados impregnam a visão pre- 
ponderantemente adotada pela formação acadêmica em Psicologia.

A pesquisa realizada por Bilgrave e Deluty (1998) sobre a relação entre a espiritualidade e as escolhas teóricas dos psicólogos conclui que, da mesma forma que a exposição aos conteúdos da psicologia interfere nas escolhas espirituais e religiosas, também a prática de determinada forma de espiritualidade, ou a participação em alguma religião instituída, interfere na escolha da orientação psicológica adotada pelos profissionais da psicologia. Os psicólogos de religiosidade cristã são apontados como mais inclinados à orientação cognitivo-comportamental, enquanto aqueles que professam sua espiritualidade sem adotar uma religião específica, pautando-se por crenças transculturais, transreligiosas, místicas e orientais, tendem a abordagens humanistas, existencialistas e suas derivações, como é o caso de abordagens mais contemporâneas, como a psicologia transpessoal. No mesmo estudo, não foram encontradas referências quanto às manifestações religiosas ou espirituais dos psicanalistas, o que enfatiza a correlação com os menores índices de bem-estar espiritual observados neste estudo.

\section{Espiritualidade na formação em Psicologia no Rio Grande do Sul}

Partindo de pesquisas publicadas nas bases de dados nacionais e internacionais, que já referem a religiosidade/espiritualidade dos psicólogos como significativamente inferior à da população em geral, buscou-se conhecer mais aprofundadamente as implicações entre a formação acadêmica em psicologia e a dimensão espiritual de futuros psicólogos. Na mesma direção dessas pesquisas, os resultados deste estudo revelam que o bem-estar espiritual dos formandos em Psicologia das universidades gaúchas é significativamente menor que o dos calouros.

O aumento da escolaridade, decorrente da formação acadêmica, já foi apontado por Marques (2000) como um dos fatores que contribuem para a diminuição do bem-estar espiritual da população em geral, pois, segundo ela, já é sabido que, no 42 contexto acadêmico, a religiosidade e a espirituali- dade, inerentes ao humano, não têm o mesmo espaço para seu desenvolvimento quando comparadas ao avanço da racionalidade.

No que se refere aos estudantes de Psicologia do Estado do Rio Grande do Sul, esse dado se confirma e parece justificado pelas abordagens teóricas predominantemente desenvolvidas durante a formação, baseando-se em postulados que coincidem com os que emergiram séculos atrás, quando da busca de legitimação científica da psicologia e questionamento das instituições religiosas. É preciso considerar que o perfil espiritual e religioso dos estudantes e dos profissionais da psicologia parece seriamente influenciado pela posição da vertente psicanalítica. Sem entrar no mérito das grandes contribuições da psicanálise, ressalta-se aqui a possibilidade de que a concepção psicanalítica de religiosidade como um aspecto relacionado à infantilidade humana ainda esteja sendo transmitida sem a devida discriminação entre religiosidade e espiritualidade, o que dificulta a legitimação desse tema em psicologia. Tal postura merece questionamento para que a irredutibilidade e o cerceamento dogmático das instituições religiosas, devidamente combatidos pela psicanálise, não acabem sendo reproduzidos em sua própria concepção diante da inteireza das dimensões humanas e, assim, da espiritualidade.

Os resultados do presente estudo, além de confirmarem a hipótese, inicialmente levantada, de que a espiritualidade de formandos seria menor que a de calouros, reforçam os achados e reflexões amplamente abordados nas publicações recentes e nas pesquisas disponíveis nas bases de dados (AnconaLopez, 2008b; Angerami-Camon, 2008; Boccalandro, 2004; Bruscagin et al., 2008; Costa et al., 2008; Erthal, 2004; Gastaud et al., 2006; Marques, 1996; Peres et al., 2007). Esses resultados nos reportam à necessidade de legitimação da espiritualidade na esfera científica e, assim, à inserção do estudo da espiritualidade na formação acadêmica em Psicologia.

Os dados apresentados indicam a necessidade de revisão e de reformulação dos paradigmas norteadores da ciência psicológica, que tem negligenciado uma dimensão inerente ao humano que interfere na saúde psíquica. O percurso vivenciado 
durante a formação acadêmica em psicologia parece estar contribuindo para o embotamento da espiritualidade dos próprios psicólogos, ao adotarem religiosamente suas doutrinas psicológicas.

Estudos acerca da espiritualidade (Amatuzzi, 1999; Marques, 2000) também relacionam o bem-estar espiritual com a necessidade de autotranscendência, ou seja, a conexão com um propósito que justifique nosso comprometimento com a vida. Esse aspecto parece inerente ao profissional que se propõe ao exercício da psicologia, independentemente de sua opção teórica. Marques (2000) corrobora a ideia de autores que afirmam haver maior bem-estar psicológico e maior probabilidade de se encontrar comportamentos de apoio aos outros em pessoas que têm uma visão espiritual positiva diante da vida, o que sugere que o desenvolvimento de sua espiritualidade pode proporcionar melhor atenção e cuidado à saúde integral de outrem. Porém, de acordo com os resultados deste estudo, o curso de psicologia parece estar contribuindo inversamente para isso, provocando a diminuição do bem-estar espiritual. Além disso, o autocentramento dogmático em suas teorias de base acaba por interferir diretamente na autotranscendência egoiga de estudantes que inicialmente optam por uma profissão que exige abertura à inteireza do outro Ser.

Sugerem-se continuadas investigações a fim de se compreender a relação entre formação acadêmica e espiritualidade para que essa grave contradição e dissociação entre a espiritualidade do psicólogo e a espiritualidade dos demais seres humanos seja transcendida.

\section{Referências}

Amatuzzi, M. M. (1999). Desenvolvimento psicológico e desenvolvimento religioso. In M. Massini \& $\mathrm{M}$. Mahfound (Orgs.), Diante do mistério: psicologia e senso religioso (pp.123-140). São Paulo: Loyola.

Ancona-Lopez, M. (1999). Religião e psicologia clínica: quatro atitudes básicas. In M. Massini \& M. Mahfound (Orgs.), Diante do mistério: psicologia e senso religioso (pp.71-86). São Paulo: Loyola.

Ancona-Lopez, M. (2008a). A religiosidade do psicoterapeuta. In C. Bruscagim, A. Savio, F. Fontes \& D. M.
Gomes (Orgs.), Religiosidade e psicoterapia (pp.1-8). São Paulo: Rocca.

Ancona-Lopez, M. (2008b). Religião na constituição da subjetividade: psicólogos e seus clientes. In V. A. Angerami (Org.), Psicologia e religião (pp.69-84). São Paulo: Cengage Learning.

Angerami-Camon, V. A. (2008). Religiosidade e psicologia: a contemporaneidade da fé religiosa nas lides acadêmicas. In V. A. Angerami (Org.), Psicologia e religião (pp.1-42). São Paulo: Cengage Learning.

Aten, J. D., \& Hernandez, B. C. (2005). A 25 year review of qualitative research publisched in spiritually and psychologically oriented journals. Journal of Psychology and Christianity, 24(3), 266-277.

Bilgrave, D. P., \& Deluty, R. H. (1998). Religious beliefs and therapeutic orientations of clinical and counseling psychologists. Journal for the Scientific Studies of Religion, 37(2), 329-349.

Boccalandro, M. P. R. (2004). Vontade: um atributo do Self. In V. A. Angerami-Camon (Org), Espiritualidade e prática clínica (pp.137-153). São Paulo: Pioneira Thomson Learning.

Bruscagin, C., Savio, A., Fontes, F., \& Gomes, D. M. (2008). Religiosidade e psicoterapia. São Paulo: Rocca.

Cerqueira, E. (2008). Comportamento sexual e religiosidade (Tese de doutorado não-publicada). Programa de Pós-Graduação em Psicologia, Universidade Federal do Rio Grande do Sul, Porto Alegre.

Costa, C. C., Bastiani, M., Geyer, J. G., Calvetti, P. U., Muller, M. C., \& Moraes, M. L. A. (2008). Qualidade de vida e bem-estar espiritual em universitários de psicologia. Psicologia em Estudo, 13(2), 249-255.

Erthal, T. C. S. (2004). A luz da sabedoria na psicoterapia. In V. A. Angerami-Camon (Org.), Espiritualidade e prática clínica (pp.1-37). São Paulo: Pioneira Thomson Learning.

Fleck, M. P. A. (2004). Religiosidade, saúde e qualidade de vida: uma revisão de literatura. In E. F. B. Teixeira, M. C. Muller, \& J. D. T. Silva (Orgs.), Espiritualidade e qualidade de vida (pp.165-180). Porto Alegre: EDIPUCRS.

Gastaud, M. B., Souza, L. D. M., Braga, L., Horta, C. L., Oliveira, F. M., Souza, P. L. R., et al. (2006). Bem-estar espiritual e transtornos psiquiátricos menores em estudantes de psicologia. Revista de Psiquiatria do Rio Grande do Sul, 28(1), 12-18.

Greeley, A. M., \& Hout, M. (1999). American's increasing belief in life after death: Religious competition and acculturation. Sociology Review, 64, 813-835.

Instituto Brasileiro de Geografia e Estatística. (2000). Censo demográfico Brasil. Brasília: IBGE.

Koenig, H. G. (2007). Religião, espiritualidade e psiquiatria: uma nova era na atenção à saúde mental. Revista de Psiquiatria Clínica, 34(1), 5-7. 
Kovács, M. J. (2007). Espiritualidade e psicologia: cuidados compartilhados. O Mundo da Saúde, 31(2), 246-255.

Lukoff, D. (2003). Problemas religiosos e espirituais. In Conferência do IV Congresso Internacional de Psicologia Transpessoal da Associação Luso Brasileira de Transpessoal. Cascais, Portugal.

Marques, L. F. (1996). Práticas alternativas em psicoterapia num cenário de mudança de paradigma. Psico, 27(1), 161-184.

Marques, L. F. (2000). A saúde e o bem-estar espiritual em adultos porto alegrenses (Tese de doutorado não-publicada). Programa de Pós-Graduação em Psicologia, Pontifícia Universidade Católica do Rio Grande do Sul, Porto Alegre.

Marques, L. F. (2003). A saúde e o bem-estar espiritual em adultos porto alegrenses. Psicologia: Ciência e Profissão, 23(2), 56-65.

Marques, L. F., Sarriera, J. C., \& Dell'Aglio, D. D. (2008). Adaptação e validação da Escala de Bem-estar Espiritual (EBE). Revista Avaliação em Psicologia, 8(2), 179-186.

Moreira-Almeida, A. M., Lotufo Neto, F., \& Koening, H. G. (2006). Religiousness and mental health: A review. Revista Brasileira de Psiquiatria, 28(3), 242-250.

Paloutzian, R. F., \& Elisson, C. W. (1982). Loneliness, Spiritual Well-being and the quality of life. In L. A.
Peplau \& D. Perlman (Orgs.), Lonelines, a sourcebook of current theory, research and theraphy (pp.224-237). New York: Wiley.

Panzini, R. G., Rocha, N. S., Bandeira, D. R., \& Fleck, M. P. A. (2007). Qualidade de vida e espiritualidade. Revista de Psiquiatria Clínica, 34(1), 105-115.

Pedrão, R. B., \& Beresin, R. B. (2009). O enfermeiro frente à questão da espiritualidade. HIAE, 8(1 Pt 1), 86-91.

Peres, J. F. P., Simão, M. J. P., \& Nasello, O. A. G. (2007) Espiritualidade, religiosidade e psicoterapia. Revista de Psiquiatria Clinica, 34(1), 136-145.

Rocca-Larrosa, S. M. (2007). Resiliência e cuidado. Revista IHU on line, 7(234), 56-59.

Sanchez, Z. D. M., \& Nappo, S. A. (2007). A religiosidade, a espiritualidade e o consumo de drogas. Revista de Psiquiatria Clínica, 34(1), 73-81.

World Health Organization Quality of Life Group. (1994). The development of de world heallth organization quality of life assessment instrument (the WHOQOL). In J. Orley \& W. Kuyken (Eds.), Quality of life assessment: International perspectives (pp.41-60). Heidelberg: Springer Verlag.

Recebido em: 15/3/2012

Aprovado em: 24/4/2012 